\title{
Knowledge Management in Academic Industry Collaborations: how to best foster Innovation Capability?
}

\section{Authors}

Dr Clare Thornley Innovation Value Institute, National University of Ireland, Maynooth, Ireland Dr Shane McLoughlin LERO (Irish Software Research Institute), National University of Ireland Maynooth, Ireland

Prof Kalpana Shankar School of Information \& Communication Studies, University College Dublin, Ireland

\begin{abstract}
Purpose

Academic industry collaborations (AIC) are increasingly seen as a key part of national innovation policies as an approach to creating and exploiting knowledge. They, however, present a complex challenge in terms of knowledge management (KM). This is partly due to the wide range of stakeholders, the input of government policy, and also the different cultures, practices and expectations of those involved. The research questions are as follows:

RQ1: How do participants perceive and experience knowledge creation and transfer in academic industry collaborations?

RQ2: How can the intersection between knowledge management literature and AIC inform and improve both disciplines and practices?
\end{abstract}

Methods

(1) An analysis of KM literature in terms of AIC. (2) Exploratory in-depth interviews with participant stakeholders of AIC projects. (3) Findings are then analysed in terms of existing concepts in KM literature.

Findings

Initial findings suggest that a more in-depth acknowledgement of the role of power, tensions and differences between stakeholders is important in understanding and improving the innovation potential of AIC projects. A more nuanced understanding of knowledge transfer is also needed with tailored approaches for particular project stages and organisations.

Practical Implications

The role of academic industry collaborations in fostering innovation by facilitating knowledge creation and transfer is seen as increasingly important as a social and political priority but KM has so far not yet engaged fully with this. This paper suggests that extending KM from a primarily organisation level focus to a broader societal focus would be a useful development.

Key words: innovation; knowledge transfer; academic industry collaboration; science policy; knowledge management.

\section{Introduction}

The question of the role that research, particularly scientific research, should play in society has been under discussion for many decades. At a fundamental level the point of science is to increase our knowledge of the world. However, the ways in which that knowledge development should be funded, managed, and the extent of state control over it, is disputed territory. The question of what knowledge should be pursued and to what and whose ends is a persistent issue in national and international knowledge and innovation policies. J.D. Bernal published 'The Social Function of Science' (Bernal, 1939) in the 1930s with his critical and masterful overview of the key issues. Bernal admitted his own bias and said he wrote from his own experience of the frustration and failures of science to work for coordinated ends. He also acknowledged the 'danger of any organisation of science destroying the originality and spontaneity of which are essential to its progress' (p. xiii), whilst ultimately believing that it is possible to combine freedom and efficiency in science. 
The role of academic industry collaboration (AIC) in fostering innovation by facilitating knowledge creation and transfer, is seen as increasingly important as a social and political priority and is well evidenced in many science and innovation policy initiatives (SFI, 2016). There is, however, limited input from KM research and theory in terms of how it works in practice and could be improved. The particular aspect of science policy we examine is the role of AIC research projects with a focus on the Irish context. We then discuss some of the issues through the lens of KM arguing that many issues in science policy and AIC projects are familiar to the KM community and that a sharing of perspectives could mutually benefit both fields. This study seeks to contribute to both KM and AIC by exploring potential linkages and mutual learnings. The research questions we discuss are as follows:

RQ1: How do participants perceive and experience knowledge creation and transfer in academic industry collaborations?

RQ2: How can the intersection between knowledge management literature and AIC inform and improve both disciplines and practices?

\section{Literature review}

In this section a brief overview of the key concepts in KM particularly relevant to academic industry collaboration (AIC) are introduced, followed by a discussion of some more recent science policy research with a focus on AIC. The objective is to illustrate how a grounding in KM concepts may help in exploring some of the diverse trajectories within the science policy debate.

\subsection{Knowledge management: key concepts}

The acknowledged tension within allowing the freedom to make knowledge creation and discovery possible whilst also wishing to manage it in such a way that it meets strategic objectives is a familiar theme within both research policy and the KM literature. With particular relevance to AIC the complexities of managing structures, processes and rewards around knowledge management that are appropriate to particular organisational needs (Kieser and Leiner, 2009; Andreeva and Kianto, 2016) and the issue of how differing cultures of communication and knowledge activities can make trust and cooperation difficult (Serenko and Bontis, 2016), are both well discussed in the KM literature.

The problem of how to best organise knowledge so as to effectively store, retrieve and communicate it is also a strong concern in science policy and in KM. Bernal (Bernal, 1939) discussed the 'burying of published work; (p. 117) as even in the 1930s he felt that information overload was a serious problem with the risk of important work being forgotten or missed and thereby not informing progress. This is discussed recently in terms of Bernal's influence on the development of the citation index by Eugene Garfield (JD Bernal: politics and science - Irish Philosophy, 2018). More recently this has become a major concern in terms of the open access movement and the requirement by funders that certain types of scholarly outputs are made freely available. This can be broadly positive in terms of access to knowledge but can be in tension with the knowledge culture and reward research system as researchers often need prestigious knowledge outputs in non-open access journals to further their careers and ensure recognition of research excellence.

A key theme in terms of the value of KM is the role it can play in effective knowledge exploitation, the actual use of knowledge by an organisation to gain benefits from its knowledge (Raisch et al., 2009). The various organisational attributes and policies that enable an organisation to gain and also exploit knowledge was termed absorptive capacity in 1990 (Cohen and Levinthal, 1990) and this influential concept has since been further refined (Zahra and George, 2002). Absorptive capacity is an important but complex factor in the success of AIC (Muscio, 2007) as if companies do not have the ability to absorb and exploit new knowledge, then simply putting them in proximity to it will not be adequate to foster or exploit innovation. This is also linked to the relationship between KM and organisational learning with considerable research on the 'correct' balance between knowledge exploration and exploitation (Gupta, Smith and Shalley, 2006).

\subsection{Models of KM in academic industry collaboration}

A consistent issue throughout the AIC literature (Perkmann, 2009; Perkmann et al., 2013) is the correct balance between funding what can be called fundamental, basic (or sometimes 'blue sky' research) and

Thornley, C; McLaughlin, S and Shankar, K. Knowledge Management in Academic Industry Collaborations: how to best foster Innovation Capability? European Conference on Knowledge Management, Lisbon, Portugal 5-6 September 2019. 
funding problem or solution 'relevant' research to address particular issues. The relationship between these two approaches to research, or more accurately two locations on the fundamental to applied research spectrum, is also unclear and disputed (Blumenthal et al., 1996; Bentley, Gulbrandsen and Kyvik, 2015). It is acknowledged that fundamental research should be supported both for 'its own sake' (i.e. to advance human knowledge) and also because it can lead to unexpected solutions to problems or highly relevant applications. It is also argued that innovation can come out of collaboration with diverse research teams, involving both industry partners and academic researchers, and focussing on 'real world' problems in this way progresses knowledge in new ways which then have clear relevance (Weckowska, 2015) and socio-economic benefits.

In terms of how AIC can be defined and conceptualised, some work has been done, with a recent structured literature review (Perkmann et al, 2013) providing a good summary of the key conceptualisations in the field including the nature of knowledge transfer. A more recent review (Rybnicek and Königsgruber, 2019) indicated the factors that influence success but suggests that more in depth research is needed to see how they operate in practice. There has also been some policy work carried out and different business models for funding innovation proposed (Ivascu et al, 2016). In terms of in-depth analysis of stakeholder perceptions and practice, there is limited qualitative work in this area. From an Irish context, the most recent research perceptions from stakeholders is more than 10 years old (Ryan et al, 2008).

\subsection{Unpacking knowledge transfer}

The role that AIC can play in innovation and knowledge creation is often termed 'knowledge transfer' (Rynes, Bartunek and Daft, 2001; Perkmann et al., 2013). This terminology, in itself, is fairly clearly a simplification of what is actually going on with knowledge in these projects (Ramos-Vielba and Fernández-Esquinas, 2012), as with knowledge transfer in any context (Wehn and Montalvo, 2018). Knowledge transfer terminology can be vague and sometimes is discussed as 'knowledge exchange' or 'knowledge diffusion' which can cause confusion (Dan and Suneson, 2012). Critical reviews of the term (Thompson, Estabrooks and Degner, 2006; Mitton et al., 2007) agree that it is simply not the case that putting knowledge in front of someone means they will be able to use it in a constructive way and ensure knowledge valorisation. This is also linked to organisational capability to deal with knowledge and absorptive capacity to exploit it as previously discussed. Clear evidence of knowledge transfers from industry to academia and vice versa is normally taken to fall under some of the impact criteria which can include publications and citations on the academic side, and patents, spin off companies, commercialisations, successful scaling, on the industry side. The literature also strongly suggests that knowledge transfer does not only occur with explicit knowledge, but also in complex ways through developing new relationships and ways of communicating between industry and academic partners (Hughes and Kitson, 2012). The role of power in AIC research and its impact on knowledge transfer is discussed in terms of Foucault's work (Ekbia and Kling, 2003; Heizmann and Olsson, 2015), arguing that knowledge is not always benignly shared for the social or organisational good as perhaps is too often assumed in much of KM literature.

\subsection{National context}

Government support for research involving industry, either at a national or EU level, is often characterised by discourse around fostering innovation and developing and maintaining competitiveness in a global environment. To this end the support of industry players (particularly SMEs and/or companies involved in the high-tech sector) are often lauded as essential. Ireland was particular badly hit by the economic crash in 2008 and the recession that followed had a major impact on public funding of scientific research and research in general. Prior to this, in an era referred to as the 'Celtic Tiger', research funding was more open, and researchers generally directed the focus and type of research they undertook. This was not seen as wholly positive with critiques of the lack of university support for research projects collaborating with industry (Ryan, Wafer and FitzGerald, 2008). There was relatively little explicit focus on the need for social and economic impact. This changed dramatically after the crash as government funding bodies became more focussed on funding research that would drive economic growth. The legacy of this shift has remained with current policy (SFI, 2016) which emphasizes the role of industry, and research that has engaged industry and produced 'real world' impact. It was within this context that one of the major funders, Science Foundation Ireland, launched their science policy funding programme of which this study is one part of.

Thornley, C; McLaughlin, S and Shankar, K. Knowledge Management in Academic Industry Collaborations: how to best foster Innovation Capability? European Conference on Knowledge Management, Lisbon, Portugal 5-6 September 2019. 


\section{Methods}

This exploratory grounded study is still in progress, but data collection has thus far yielded 16 semi-structured qualitative interviews with a mixture of academic researchers; industry partners and research policy experts. All participants were selected based on having experience working within at least one AIC. Questions included prompts to cover participant's experience and perceptions in an exploratory fashion, including the key topics of: relationships and culture; research impact; knowledge creation and transfer; science and innovation policy; enablers and blockers to successful collaboration. It was explained to participants that the aim is to improve our understanding of how AIC research projects can better enable innovation, knowledge creation \& transfer, so that appropriate state supports to select, support and monitor such programmes can be improved. For this paper, transcripts were thematically analysed by the researcher to identify points strongly related to KM including knowledge transfer, innovation policy, ethics, knowledge communication and knowledge cocreation.

Table 1: Research Participants

\begin{tabular}{|l|l|l|l|}
\hline Career stage & gender & Subject & Sector \\
\hline advanced & $\mathrm{M}$ & IS/IT/anthropology & University \\
\hline early & $\mathrm{F}$ & pharmaceuticals & University \\
\hline very early & $\mathrm{F}$ & $\mathrm{KM} / \mathrm{IS} /$ community engagement & University \\
\hline late & $\mathrm{M}$ & $\mathrm{IT} /$ engineering & University \\
\hline medium & $\mathrm{M}$ & academic/ethics & Policy expert \\
\hline advanced & $\mathrm{M}$ & Research Centre Funding Manager & Policy expert \\
\hline late & $\mathrm{M}$ & Business/MIS/Psychology & University \\
\hline advanced & $\mathrm{F}$ & Medical Imaging/Information Science & University \\
\hline medium & $\mathrm{M}$ & mathematics & University \\
\hline advanced & $\mathrm{M}$ & Information science & Industry (small) \\
\hline mid & $\mathrm{M}$ & financial IT & Industry (large) \\
\hline advanced & $\mathrm{M}$ & business/ IT. software & University \\
\hline advanced & $\mathrm{M}$ & Business/IT & University \\
\hline advanced & $\mathrm{M}$ & IT/ engineering & Industry (large) \\
\hline advanced & $\mathrm{M}$ & IT/ engineering & University \\
\hline advanced & $\mathrm{M}$ & IT/health & Industry (large) \\
\hline
\end{tabular}

\section{Findings}

In this section we describe the data from the interviews through the lens of knowledge management. These findings are discussed in terms of key themes that emerged from the data that strongly relate to Knowledge Management concepts and models.

\subsection{Knowledge Strategy}

Within the KM literature, the alignment of KM strategy with the needs and nature of the organisation is seen as key success factor (Oluikpe, 2012; Andreeva and Kianto, 2016) and this alignment issue was also a strong theme in the data in terms of the importance of science policy being closely tied to the capacity and needs of individual countries. A strong recurring theme was that further and careful thought should inform which science and innovation areas best produces scientific contributions and socio-economic value for a country when considering its characteristics and size. It was felt by the majority of participants that this strategic high level foresight activity can too easily be entwined with the nuts and bolts of research and that this needs to be clearly separate. In terms of structures, those making decisions about a national research strategic priority should not be too close to the research institutions doing the work. The most effective development of new knowledge has to be strategically linked to the external environment and the strengths of the country in terms of skills, size, geography and existing research expertise. Within Ireland and the UK participants, there was limited confidence in existing structures for industry or academics to influence government priorities for

Thornley, C; McLaughlin, S and Shankar, K. Knowledge Management in Academic Industry Collaborations: how to best foster Innovation Capability? European Conference on Knowledge Management, Lisbon, Portugal 5-6 September 2019. 
research funding. However, in the broader European context it was generally agreed that industry had fairly good mechanisms to feed into and inform EU research priorities.

\subsection{Power and knowledge}

The formulation of science policy, particularly when it involves industry input, clearly involves large sums of money and the ability to control access to research funds was seen as problematic by some participants. One participant questioned, 'Are funders really benign, they have enormous power?' Often the same people who are on funding review panels are on influential journal boards, and thus one's career trajectory could be affected by any negative interactions with members. Industry funders also will have influence based on their economic contribution to projects and it can be hard to ascertain how this may influence research priorities. The risks for the different players in AIC projects are all very different depending on how much power they have or how securely their prestige is embedded in their context of work. A PhD student, for example, is at more risk in a collaborative project as he or she needs to meet some very defined criteria in terms of 'original contribution to knowledge' to obtain a PhD. Another observation from an early career female researcher was how her knowledge was only seen as legitimate when introduced by a senior Professor, and it was noted that if Professors did not support or validate their younger colleagues in this way then often their knowledge contributions were ignored or down played.

Interestingly, some of the most senior participants, whist being aware their careers and prestige were secure, mentioned that the push for research with industry was so tempting in terms of funds and prestige that it did result in their original research direction and interests being redirected almost without being fully aware of it.

\subsection{Types of knowledge}

The definition of knowledge and a conceptualisation of the different types of knowledge is a perennial issue in the KM literature (Alavi and Leidner, 2007; Stehr and Grundmann, 2011). One key issue that emerged from the interviews was the diversity and complexity of types and stages of knowledge which existed within AIC. Within industry, for example, a new process can be as important or more important than a new scientific discovery. It can enable more affordable manufacture, be transferable to another product, or just enable scaling up of an existing product. Knowledge of how to do a process, for example, is fundamentally different in terms of how it can be shared and transferred than for example, knowledge of a new product which is much more restricted in terms of patents etc. The different stages of knowledge development were also seen as important, so exploratory stages require different management than the solution-focussed stage. In terms of knowledge outputs, it was observed that often this is simply divided into industry output and academic output and that the complex differences within these areas can be conflated. Within the academic context, for example, developing a paper for a prestigious journal is significantly different from developing an output for a mid or lower tier journal. In terms of the former there are very specific parts of the research that need to be in place in terms of model, method and research subjects which may not sit easily with industry partners or other requirements of the research project. Thus, generic guidance on 'how to' publish with industry were often not useful as they did not reflect a deep understanding of the requirements of high-level academic publishing. This was also seen as true in the very specific nature of research qualifications. For example, knowledge concerned with gaining a PhD cannot easily be mixed up with other types of knowledge in the project without a level of risk that academic criteria will not be met.

\subsection{Knowledge creation}

The overall aim of AIC is to foster innovation and knowledge creation and transfer. In terms of this project, one of the aims was to ascertain how current approaches to AIC could be improved to make this a more likely outcome.

The engagement of different stakeholders in research, often termed the co-creation of knowledge, is a growing research practice. This is seen, for example, in recent work on smart cities, a research area requiring at the least; collaboration between technology developers, local government and local residents (Tokoro, 2016). The best way to manage this process generated considerable discussion with participants. One important question, linked to the previous discussion of power, was who gets asked to join in these networks? SMEs, for instance, can often be under represented, so important insights from smaller companies are lost. In

Thornley, C; McLaughlin, S and Shankar, K. Knowledge Management in Academic Industry Collaborations: how to best foster Innovation Capability? European Conference on Knowledge Management, Lisbon, Portugal 5-6 September 2019. 
another example a participant made the case that academic researchers could certainly have already come up with the correct relevant research areas to prioritise without industry input. The engagement of industry stakeholders throughout the process, however, had created relationships and networks that would be of value. In this case useful knowledge was not really being transferred, as awareness was already high, but consultation brought benefits that were likely to be useful at a later stage.

Stages of knowledge development was also seen as important and stakeholder engagement should and did change with time. In some cases, industry can have their own ideation process then later bring in academic experts to advise on detail. Conversely, in an example from health IT, the company had used academic experts to shape direction, then leveraged focussed researchers (some industrial and some academic) to deliver solutions. This distinction between different types of stakeholder input at different stages of the knowledge creation process is important as it suggests that support around knowledge creation needs to be an adaptive process rather than one approach for the whole project. Co-creation was not only seen as a social good but often a business imperative as customer loyalty is now more fragile and fickle.

An interesting insight that came up was that the requirement for co-creation and joint working was not always supported by adequate skill or capacity development between the different participants. It was observed that often communication processes were put in place, such as weekly meetings or stakeholder communication events, but that communication and effective knowledge transfer often did not happen because the expectation, needs and vocabulary of the different participants were too diverse. There was also a mismatch between the industry timeline wanting definite answers and academics, in particular PhD students, whose work at early stages was nearly always diffuse. Knowledge exchange did not actually happen if the structure of the communication process was inappropriate. In order for it to work, industry partners really needed to take the time to understand the academics' viewpoint and vice versa.

\subsection{The nature of expertise}

The ability to accurately locate and recognise appropriate experts is seen as key part of effective KM (Stehr and Grundmann, 2011; Dieng-Kuntz and Matta, 2012). In terms of AIC, this seemed to pose two major challenges. Firstly, the problem of correctly identifying an appropriate expert and secondly, knowing how to verify their expertise. For the former problem, it was considered that government funding bodies have considerable knowledge about identifying experts on particular topics to review projects for funding but that this knowledge is not effectively shared. Industry has to start from its own contacts to identify research experts. Secondly, it was observed that there is a significant difference in how industry expertise and academic expertise are publically verifiable. In terms of an academic researcher, publications and citations offer a visible and verified indicator of expertise. For industry experts, outputs may not always be published or easy to verify by those outside the industry. In terms of selecting experts to sit on review committees or panels it can be difficult to optimise the blend of expertise. A quota mentality was perceived to exist so there could be an apparent diversity of roles (e.g. one academic, one senior industry, one SME) but not necessarily a diversity of ways of thinking.

\section{Discussion}

The emerging themes are interesting in terms of knowledge management as many are familiar and they reflect some of the tensions and trade-offs core to the discipline and practice of managing something as complex and multi-faceted as knowledge. Key themes that have been discussed in the previous section: knowledge strategy; power and knowledge; knowledge transfer; types of knowledge; knowledge creation; the nature of expertise, are all key aspects of KM and we argue that KM could make a useful contribution to improving how these are managed within AIC. Additionally, KM could benefit from exploring the rich context of knowledge within these collaborations and their role in innovation policies.

Even at the most fundamental level of the distinction between data, information and knowledge; we see plenty of evidence for Tuomi's (Tuomi, 1999) assertion that some shared knowledge or culture must be in place before data and information exchange can meaningfully take place. Communicating knowledge across different groups with different cultures and ways of working is well known to be challenging (Loewenstein,

Thornley, C; McLaughlin, S and Shankar, K. Knowledge Management in Academic Industry Collaborations: how to best foster Innovation Capability? European Conference on Knowledge Management, Lisbon, Portugal 5-6 September 2019. 
Ocasio and Jones, 2012; Ferguson et al., 2017) and the concept of different vocabularies of knowledge providing a barrier to communication are well evidenced in the findings of our research.

\section{Conclusions and future work}

Our findings so far present a reasonable case that $\mathrm{KM}$ as a discipline should be more engaged with science and innovation policy to address the complex problem of bringing different groups together to create knowledge for wider social benefits. In terms of our research questions we can conclude the participants have complex experiences of knowledge in AIC and that KM concepts and models can usefully inform the nature of these complexities. AIC can also provide fertile ground for improving and refining KM as a practice and discipline. $\mathrm{KM}$ at present is generally focussed on the micro level of the organisation rather than the wider social and political sphere. Should science and innovation policy be seen as part of KM? Given the current focus on innovation and the increased requirement that the knowledge created must be developed and have impact in a range of new and diverse ways; this could be an excellent opportunity for KM to find a new field of influence.

\section{References}

Alavi, M. and Leidner, D. E. (2007) 'Review: Knowledge Management and Knowledge Management Systems: Conceptual Foundations and Research Issues', MIS Quarterly, 25(1), pp. 107-136.

Andreeva, T. and Kianto, A. (2016) 'Empirically Testing the Role of Strategic Management of Knowledge in Firm Performance', in European Conference of Knowledge Management. Belfast.

Bentley, P. J., Gulbrandsen, M. and Kyvik, S. (2015) 'The relationship between basic and applied research in universities', Higher Education, 70(4), pp. 689-709.

Bernal John Desmond (1939) The Social Function of Science. London: Routledge.

Blumenthal, D. et al. (1996) 'Relationships between Academic Institutions and Industry in the Life Sciences An Industry Survey', New England Journal of Medicine, 334(6), pp. 368-374.

Cohen, W. M. and Levinthal, D. A. (1990) 'Absorptive Capacity: A New Perspective on Learning and Innovation', Administrative Science Quarterly, 35(1), pp. 128-152

Dan, P. and Suneson, K. (2012) 'Knowledge Transfer, Knowledge Sharing and Knowledge Barriers-Three Blurry Terms in KM', The Electronic Journal of Knowledge Management, 10(1), pp. 81-91.

Dieng-Kuntz, R. and Matta, N. (2012) Knowledge management and organizational memories. Springer Science and Business Media.

Ekbia, H. R. and Kling, R. (2003) 'Power in Knowledge Management in late Modern Times', Academy of Management Proceedings, 2003(1), pp. D1-D6.

Ferguson, J. E. et al. (2017) 'Structure, Content, and Meaning of Organizational Networks: Extending Network Thinking, Introduction', in Structure, Content and Meaning of Organizational Networks, pp. 1-15.

Gupta, A. K., Smith, K. G. and Shalley, C. E. (2006) 'The Interplay Between Exploration and Exploitation', Academy of Management Journal, 49(4), pp. 693-706.

Heizmann, H. and Olsson, M. R. (2015) 'Power matters: the importance of Foucault's power/knowledge as a conceptual lens in KM research and practice', Journal of Knowledge Management, 19(4), pp. 756-769.

Hughes, A. and Kitson, M. (2012) 'Pathways to impact and the strategic role of universities: New evidence on the breadth and depth of university knowledge exchange in the UK and the factors constraining its development', Cambridge Journal of Economics, 36(3), pp. 723-750.

JD Bernal: politics and science - Irish Philosophy (2018). Available at: http://www.irishphilosophy.com/2018/05/10/bernal-politics-science/ (Accessed: 28 March 2019).

Kieser, A. and Leiner, L. (2009) 'Why the Rigour - Relevance Gap in Management Research Is Unbridgeable', Journal of Management Studies, 46(3), pp. 516-533.

Loewenstein, J., Ocasio, W. and Jones, C. (2012) 'Vocabularies and Vocabulary Structure: A New Approach Linking Categories, Practices, and Institutions', The Academy of Management Annals, 6(1), pp. 41-86.

Mitton, C. et al. (2007) 'Knowledge transfer and exchange: review and synthesis of the literature.', The Milbank quarterly, 85(4), pp. 729-68.

Muscio, A. (2007) 'The Impact of Absorptive Capacity n SMEs Collaboration', Economics of Innovation and New Technology, 16(8), pp. 653-668.

Oluikpe, P. (2012) 'Developing a corporate knowledge management strategy', Journal of Knowledge Management. Emerald Group Publishing Limited, 16(6), pp. 862-878.

Thornley, C; McLaughlin, S and Shankar, K. Knowledge Management in Academic Industry Collaborations: how to best foster Innovation Capability? European Conference on Knowledge Management, Lisbon, Portugal 5-6 September 2019. 
Perkmann, M. (2009) 'The two faces of collaboration: impacts of university-academic relations on public research', Industrial and Corporate Change.

Perkmann, M. et al. (2013) 'Academic engagement and commercialisation: A review of the literature on university-industry relations', Research Policy. Elsevier B.V., 42(2), pp. 423-442.

Raisch, S. et al. (2009) 'Organizational Ambidexterity: Balancing Exploitation and Exploration for Sustained Performance', Organization Science, 20(4), pp. 685-695.

Ramos-Vielba, I. and Fernández-Esquinas, M. (2012) 'Beneath the tip of the iceberg: Exploring the multiple forms of university-industry linkages', Higher Education, 64(2), pp. 237-265.

Ryan, J. G., Wafer, B. and FitzGerald, M. (2008) 'University - Industry collaboration: An issue for Ireland as an economy with high dependence on academic research', Research Evaluation, 17(4), pp. 294-302.

Rybnicek, R. and Königsgruber, R. (2019) 'What makes industry-university collaboration succeed? A systematic review of the literature', Journal of Business Economics, 89(2), pp. 221-250.

Rynes, S. L., Bartunek, J. M. and Daft, R. L. (2001) 'Across the Great Divide: Knowledge Creation and Transfer Between Practitioners and Academics', Academy of Management Journal, 44(2), pp. 340-355.

Serenko, A. and Bontis, N. (2016) 'Understanding counterproductive knowledge behavior: antecedents and consequences of intra-organizational knowledge hiding', Journal of Knowledge Management, 20(6), pp. 11991224.

SFI (2016) 'Annual Report and Accounts 2016', p. 156. doi: 10.1179/pma.2006.40.2.432.

Stehr, N. and Grundmann, R. (2011) Experts: the knowledge and power of expertise. Routledge.

Swan, J. et al. (1999) 'Knowledge management and innovation: networks and networking', Journal of Knowledge Management, 3(4), pp. 262-275.

Thompson, G. N., Estabrooks, C. A. and Degner, L. F. (2006) 'Clarifying the concepts in knowledge transfer: a literature review', Journal of Advanced Nursing, 53(6), pp. 691-701.

Tokoro, N. (2016) The Smart City and the Co-creation of Value. Tokyo: Springer Japan (SpringerBriefs in Business).

Tuomi, I. (1999) 'Data Is More Than Knowledge: Implications of the Reversed Knowledge Hierarchy for Knowledge Management and Organizational Memory', Journal of Management Information Systems. IEEE Comput. Soc, 16(3), pp. 103-117.

Weckowska, D. M. (2015) 'Learning in university technology transfer offices: transactions-focused and relations-focused approaches to commercialization of academic research', Technovation, 41-42, pp. 62-74.

Wehn, U. and Montalvo, C. (2018) 'Knowledge transfer dynamics and innovation: Behaviour, interactions and aggregated outcomes', Journal of Cleaner Production, 171, pp. S56-S68.

Zahra, S. A. and George, G. (2002) 'Absorptive Capacity: A Review, Reconceptualization, and Extension', Academy of Management Review, 27(2), pp. 185-203.

Zhang, B. and Wang, X. (2017) 'Collaboration on research performance and moderating effect of social capital: evidence from engineering academics in China', Scientometrics. 113(1), pp. 257-277.

Thornley, C; McLaughlin, S and Shankar, K. Knowledge Management in Academic Industry Collaborations: how to best foster Innovation Capability? European Conference on Knowledge Management, Lisbon, Portugal 5-6 September 2019. 